\title{
Generalized Sasakian-space-forms and Ricci almost solitons with a conformal killing vector field
}

\author{
Shyamal Kumar Hui ${ }^{1}$ and Debabrata Chakraborty ${ }^{2}$ \\ ${ }^{1}$ Department of Mathematics, Bankura University, Bankura, West Bengal, India \\ ${ }^{2}$ Department of Mathematics, Sidho Kanho Birsha University, Purulia, West Bengal, India
}

Received: 26 January 2016, Accepted: 11 July 2016

Published online: 13 August 2016.

\begin{abstract}
The present paper deals with the study of generalized Sasakian-space-forms whose metric is Ricci almost soliton with a conformal killing vector field. We obtain sufficient conditions of such type of Ricci almost solitons to be expanding, steady and shrinking respectively.
\end{abstract}

Keywords: generalized Sasakian-space-form, Ricci almost soliton, conformal killing vector field

\section{Introduction}

It is well known that in differential geometry the curvature of a Riemannian manifold plays an important role and the sectional curvatures of a manifold determine the curvature tensor $R$ completely. A Riemannian manifold with constant sectional curvature $c$ is called a real-space-form and its curvature tensor $R$ satisfies the condition

$$
R(X, Y) Z=c\{g(Y, Z) X-g(X, Z) Y\}
$$

Models for these spaces are the Euclidean spaces $(c=0)$, the spheres $(c>0)$ and the hyperbolic spaces $(c<0)$.

In contact metric geometry, a Sasakian manifold with constant $\phi$-sectional curvature is called Sasakian-space-form and the curvature tensor of such a manifold is given by

$$
\begin{aligned}
R(X, Y) Z= & \frac{c+3}{4}\{g(Y, Z) X-g(X, Z) Y\}+\frac{c-1}{4}\{g(X, \phi Z) \phi Y-g(Y, \phi Z) \phi X+2 g(X, \phi Y) \phi Z\} \\
& +\frac{c-1}{4}\{\eta(X) \eta(Z) Y-\eta(Y) \eta(Z) X+g(X, Z) \eta(Y) \xi-g(Y, Z) \eta(X) \xi\} .
\end{aligned}
$$

These spaces can also be modeled depending on $c>-3, c=-3$ or $c<-3$.

As a generalization of Sasakian-space-form, in [1] Alegre, Blair and Carriazo introduced and studied the notion of generalized Sasakian-space-form with the existence of such notion by several interesting examples. An almost contact metric manifold $M(\phi, \xi, \eta, g)$ is called a generalized Sasakian-space-form if there exist $f_{1}, f_{2}, f_{3} \in C^{\infty}(M)$, the ring of 
smooth functions on $M$, such that [1]

$$
\begin{aligned}
R(X, Y) Z= & f_{1}\{g(Y, Z) X-g(X, Z) Y\}+f_{2}\{g(X, \phi Z) \phi Y-g(Y, \phi Z) \phi X+2 g(X, \phi Y) \phi Z\} \\
& +f_{3}\{\eta(X) \eta(Z) Y-\eta(Y) \eta(Z) X+g(X, Z) \eta(Y) \xi-g(Y, Z) \eta(X) \xi\}
\end{aligned}
$$

for all vector fields $X, Y, Z$ on $M$, where $R$ is the curvature tensor of $M$ and such a manifold of dimension $(2 n+1), n>1$ (the condition $n>1$ is assumed throughout the paper), is denoted by $M^{2 n+1}\left(f_{1}, f_{2}, f_{3}\right)$.

In particular, if $f_{1}=\frac{c+3}{4}, f_{2}=f_{3}=\frac{c-1}{4}$ then the generalized Sasakian-space-forms turn out to the notion of Sasakian-space-forms. But it is to be noted that generalized Sasakian-space-forms are not merely generalization of Sasakian-space-forms. It also contains a large class of almost contact manifolds. For example, it is known that any three dimensional $(\alpha, \beta)$-trans Sasakian manifold with $\alpha, \beta$ depending on $\xi$ is a generalized Sasakian-space-form [3]. However, we can find generalized Sasakian-space-forms with non-constant functions and arbitrary dimensions.

The generalized Sasakian-space-forms have been studied by several authors such as Alegre and Carriazo ([2], [3], [4]), Belkhelfa, Deszcz and Verstraelen [9], Carriazo [12], Ghefari, Al-Solamy and Shahid [16], Gherib et. al ([17], [18]), Hui et. al ([24], [25], [38]), Kim [27], Maralabhavi and Shivaprasanna [28], Narain, Yadav and Dwivedi [30], Olteanu [31], Shukla and Chaubey [40], Yadav, Suthar and Srivastava [43] and many others.

In 1982, Hamilton [19] introduced the notion of Ricci flow to find a canonical metric on a smooth manifold. Then Ricci flow has become a powerful tool for the study of Riemannian manifolds, especially for those manifolds with positive curvature. Perelman ([33], [34]) used Ricci flow and its surgery to prove Poincare conjecture. The Ricci flow is an evolution equation for metrics on a Riemannian manifold defined as follows:

$$
\frac{\partial}{\partial t} g_{i j}(t)=-2 R_{i j}
$$

A Ricci soliton emerges as the limit of the solutions of the Ricci flow. A solution to the Ricci flow is called Ricci soliton if it moves only by a one parameter group of diffeomorphism and scaling. A Ricci soliton $(g, V, \lambda)$ on a Riemannian manifold $(M, g)$ is a generalization of an Einstein metric such that [20]

$$
£_{V} g+2 S+2 \lambda g=0
$$

where $S$ is the Ricci tensor, $£_{V}$ is the Lie derivative operator along the vector field $V$ on $M$ and $\lambda$ is a real number. The Ricci soliton is said to be shrinking, steady and expanding according as $\lambda$ is negative, zero and positive respectively.

During the last two decades, the geometry of Ricci solitons has been the focus of attention of many mathematicians. In particular, it has become more important after Perelman applied Ricci solitons to solve the long standing Poincare conjecture posed in 1904. In [39] Sharma studied the Ricci solitons in contact geometry. Thereafter Ricci solitons in contact metric manifolds have been studied by various authors such as Bagewadi et. al ([5], [6], [7], [26]), Bejan and Crasmareanu [8], Blaga [10], Chandra et. al [13], Chen and Deshmukh [14], Deshmukh et. al [15], He and Zhu [21], Hui et. al ([22], [23]), Nagaraja and Premalatta [29], Tripathi [42] and many others.

Recently Pigola et. al [35] introduced the notion of Ricci almost soliton and it can be defined on generalized Sasakian-space-form $M^{2 n+1}\left(f_{1}, f_{2}, f_{3}\right)$, if the relation (4) holds for smooth function $\lambda$ on $M^{2 n+1}\left(f_{1}, f_{2}, f_{3}\right)$.

Motivated by the above studies the object of the present paper is to study Ricci almost soliton on generalized 
Sasakian-space forms $M^{2 n+1}\left(f_{1}, f_{2}, f_{3}\right)$. The paper is organized as follows. Section 2 is concerned with preliminaries. Section 3 is devoted to the study of generalized Sasakian-space-forms whose metric tensor is Ricci almost soliton such that the potential vector field is conformal killing and obtain the sufficient conditions of such type of Ricci solitons to be expanding, steady and shrinking.

A Riemannian manifold is said to be Ricci symmetric if its Ricci tensor $S$ of type $(0,2)$ satisfies $\nabla S=0$, where $\nabla$ denotes the Riemannian connection. During the last six decades, the notion of Ricci symmetry has been weakened by many authors in several ways to a different extent such as Ricci recurrent manifold [32], Ricci semisymmetric manifold [41] etc. A generalized Sasakian-space-form $M^{2 n+1}\left(f_{1}, f_{2}, f_{3}\right)$ is said to be Ricci semisymmetric [41] if it satisfies $R(X, Y) \cdot S=0$, where $R(X, Y)$ is the curvature operator and $S$ is the Ricci tensor of type $(0,2)$. Every Ricci symmetric manifold is Ricci semisymmetric but not conversely [41]. However, in this paper it is proved that if $(g, V, \lambda)$ is a Ricci almost soliton on a generalized Sasakian-space-form $M^{2 n+1}\left(f_{1}, f_{2}, f_{3}\right)$, where $V$ is conformal killing vector field then $M^{2 n+1}\left(f_{1}, f_{2}, f_{3}\right)$ is Ricci semisymmetric if and only if it is Ricci symmetric.

In 1970 Pokhariyal and Mishra [36] were introduced new tensor fields, called $W_{2}$ and $E$ tensor fields, in a Riemannian manifold and studied their properties. According to them a $W_{2}$-curvature tensor on a generalized Sasakian-space-form $M^{2 n+1}\left(f_{1}, f_{2}, f_{3}\right), n>1$, is defined by [36]

$$
W_{2}(X, Y) Z=R(X, Y) Z+\frac{1}{2 n}[g(X, Z) Q Y-g(Y, Z) Q X],
$$

where $Q$ is the Ricci-operator, i.e., $g(Q X, Y)=S(X, Y)$ for all $X, Y$. Thereafter the $W_{2}$-curvature tensor have also been studied by various authors in different contexts. In this connection it is mentioned that in [25] Hui and Sarkar studied $W_{2}-$ curvature tensor field in a generalized Sasakian-space-form. A generalized Sasakian-space-form $M^{2 n+1}\left(f_{1}, f_{2}, f_{3}\right)$ is called $W_{2}$-flat if $W_{2}(X, Y) Z$ vanishes identically for all $X, Y$ and $Z$. In section 3, we obtain that if $(g, V, \lambda)$ is a Ricci almost soliton on a generalized Sasakian-space-form $M^{2 n+1}\left(f_{1}, f_{2}, f_{3}\right)$ with $V$ is conformal killing vector field then $M^{2 n+1}\left(f_{1}, f_{2}, f_{3}\right)$ is $W_{2}$-flat.

\section{Preliminaries}

A $(2 n+1)$-dimensional smooth manifold $M$ is said to be an almost contact metric manifold [11] if there exist an (1,1) tensor field $\phi$, a vector field $\xi$, an 1-form $\eta$ and a Riemannian metric $g$ on $M$ such that

$$
\begin{gathered}
\phi^{2}(X)=-X+\eta(X) \xi, \phi \xi=0, \\
\eta(\xi)=1, g(X, \xi)=\eta(X), \eta(\phi X)=0, \\
g(\phi X, \phi Y)=g(X, Y)-\eta(X) \eta(Y), \\
g(\phi X, Y)=-g(X, \phi Y)
\end{gathered}
$$

for any vector fields $X$ and $Y$ on $M$. An almost contact metric manifold $M$ equipped with an almost contact metric structure $(\phi, \xi, \eta, g)$ is said to be a Sasakian manifold [11] if

$$
\left(\nabla_{X} \phi\right)(Y)=\eta(Y) X-g(X, Y) \xi
$$


for any vector fields $X$ and $Y$ on $M$, where $\nabla$ is the Levi-Civita connection of $M$.

For an almost contact metric manifold $M$, a $\phi$-section of $M$ at $p \in M$ is a section $\pi \subseteq T_{p} M$ spanned by a unit vector $X_{p}$ orthogonal to $\xi_{p}$ and $\phi X_{p}$. The $\phi$-sectional curvature of $\pi$ is defined by $K(X \wedge \phi X)=g(R(X, \phi X) \phi X, X)$. A Sasakian manifold with constant $\phi$-sectional curvature $c$ is called a Sasakian-space-form and it is denoted by $M(c)$.

By virtue of (7) it follows from (3) that

$$
R(X, Y) \xi=\left(f_{1}-f_{3}\right)[\eta(Y) X-\eta(X) Y]
$$

Also from (3) we get

$$
S(X, Y)=\left(2 n f_{1}+3 f_{2}-f_{3}\right) g(X, Y)-\left\{3 f_{2}+(2 n-1) f_{3}\right\} \eta(X) \eta(Y) .
$$

Definition 1. [37] A vector field $V$ in a generalized Sasakian-space-form $M^{2 n+1}\left(f_{1}, f_{2}, f_{3}\right)$ is said to be a conformal killing vector field if it satisfies

$$
\left(£_{V} g\right)(Y, Z)=2 \rho g(Y, Z)
$$

for any vector fields $Y, Z$ on $M^{2 n+1}\left(f_{1}, f_{2}, f_{3}\right)$, where $\rho$ is a smooth function on $M^{2 n+1}\left(f_{1}, f_{2}, f_{3}\right)$ and $£$ is the operator of Lie differentiation. In particular, if $\rho$ is constant then $V$ is called homothetic and if $\rho=0$ then $V$ is called isometric as well as killing vector field.

\section{Ricci almost solitons on generalized Sasakian-space-forms}

This section deals with the study of of generalized Sasakian-space-forms whose metric is Ricci almost soliton with a conformal killing vector field and we prove the following:

Theorem 1. Let $(g, V, \lambda)$ be a Ricci almost soliton on a generalized Sasakian-space-form $M^{2 n+1}\left(f_{1}, f_{2}, f_{3}\right)$. If $V$ is conformal killing vector field then the followings are eqivalent:

(i) $M^{2 n+1}\left(f_{1}, f_{2}, f_{3}\right)$ is Einstein.

(ii) $\lambda+\rho$ is constant though $\lambda$ and $\rho$ are smooth functions.

(iii) $M^{2 n+1}\left(f_{1}, f_{2}, f_{3}\right)$ is Ricci symmetric.

(iv) $M^{2 n+1}\left(f_{1}, f_{2}, f_{3}\right)$ is Ricci semisymmetric.

Proof. Since $(g, V, \lambda)$ is a Ricci almost soliton on a generalized Sasakian-space-form $M^{2 n+1}\left(f_{1}, f_{2}, f_{3}\right)$ with $V$ is conformal killing vector field then by virtue of (13) we obtain from (4) that

$$
S(X, Y)=-(\lambda+\rho) g(X, Y)
$$

which implies that the manifold under consideration is Einstein, i.e. (i) holds and hence $\lambda+\rho$ is always constant by Bianchi's identity, though $\lambda$ and $\rho$ are smooth functions, i.e. (ii) holds.

As $M^{2 n+1}\left(f_{1}, f_{2}, f_{3}\right)$ is Einstein, its Ricci tensor is parallel, i.e. $M^{2 n+1}\left(f_{1}, f_{2}, f_{3}\right)$ is Ricci symmetric, which implies (iii). Again in [17] it is proved that if the Ricci tensor of a generalized Sasakian-space-form $M^{2 n+1}\left(f_{1}, f_{2}, f_{3}\right)$ with $f_{1} \neq f_{3}$ is parallel then $M^{2 n+1}\left(f_{1}, f_{2}, f_{3}\right)$ is Einstein, i.e. (iii) implies (i). 
It is known that every Ricci symmetric manifold is Ricci semisymmetric but not conversely [41]. Now for any $X, Y, Z, U$ on $M^{2 n+1}\left(f_{1}, f_{2}, f_{3}\right)$, we have

$$
(R(X, Y) \cdot S)(Z, U)=-S(R(X, Y) Z, U)-S(Z, R(X, Y) U)
$$

Using (14) in (15), we obtain

$$
(R(X, Y) \cdot S)(Z, U)=(\lambda+\rho)[g(R(X, Y) Z, U)+g(Z, R(X, Y) U)]=0,
$$

which implies that the manifold under consideration is Ricci semisymmetric, i.e. (iv) holds.

Now from (12) and (14), we get

$$
\begin{gathered}
-(\lambda+\rho)=2 n f_{1}+3 f_{2}-f_{3}, \\
3 f_{2}+(2 n-1) f_{3}=0 .
\end{gathered}
$$

From (17) and (18) we obtain $\lambda=-\left[\rho+2 n\left(f_{1}-f_{3}\right)\right]$.

This leads to the following:

Theorem 2. In a generalized Sasakian-space-form $M^{2 n+1}\left(f_{1}, f_{2}, f_{3}\right)$, a Ricci almost soliton $(g, V, \lambda)$ with $V$ is a conformal killing vector field is (i) shrinking for $\rho+2 n\left(f_{1}-f_{3}\right)>0$, (ii) steady for $\rho+2 n\left(f_{1}-f_{3}\right)=0$, and (iii) expanding for $\rho+2 n\left(f_{1}-f_{3}\right)<0$ respectively.

Corollary 1. A Ricci almost soliton $(g, V, \lambda)$, where $V$ is killing vector field in a generalized Sasakian-space-form, is shrinking, steady and expanding according as $\left(f_{1}-f_{3}\right)>0,=0$ and $<0$, respectively.

Corollary 2. A Ricci almost soliton $(g, V, \lambda)$, where $V$ is a killing vector field in a Sasakian-space-form, is always shrinking.

In [25] the first author and Sarkar studied $W_{2}$-curvature tensor field in a generalized Sasakian-space-form and they found

Theorem 3. [25] Every generalized Sasakian-space-form $M^{2 n+1}\left(f_{1}, f_{2}, f_{3}\right)$ is $W_{2}$-flat if and only if $3 f_{2}+(2 n-1) f_{3}=0$.

So by virtue of (18) and Theorem 3.3, we can state the following:

Theorem 4. If $(g, V, \lambda)$ is a Ricci almost soliton on a generalized Sasakian-space-form $M^{2 n+1}\left(f_{1}, f_{2}, f_{3}\right)$, where $V$ is conformal killing vector field, then $M^{2 n+1}\left(f_{1}, f_{2}, f_{3}\right)$ is $W_{2}$-flat.

\section{Conclusion}

In 2011 Pigola et. al [35] introduced the Ricci almost soliton. A Ricci almost soliton $(g, V, \lambda)$ on a Riemannian manifold $(M, g)$ satisfies the relation (4) for some smooth function $\lambda$ on $M$. In this paper we study Ricci almost solitons on generalized Sasakian-space-forms $M^{2 n+1}\left(f_{1}, f_{2}, f_{3}\right)$ such that the potential vector field $V$ is conformal killing and obtained some equivalent conditions. Also we found the conditions of Ricci almost solitons on $M^{2 n+1}\left(f_{1}, f_{2}, f_{3}\right)$ to be shrinking, steady and expanding respectively.

In 1970 Pokhariyal and Mishra [36] were introduced the $W_{2}$-curvature tensor field. In this paper it is shown that if $(g, V, \lambda)$ is Ricci almost soliton on a $M^{2 n+1}\left(f_{1}, f_{2}, f_{3}\right)$ with a conformal killing vector field then $M^{2 n+1}\left(f_{1}, f_{2}, f_{3}\right)$ is $W_{2}$-flat. 


\section{Acknowledgements}

The authors wish to express their sincere thanks and gratitude to the referees for their valuable suggestions towards the improvement of the paper.

\section{Competing Interests}

The authors declare that they have no competing interests.

\section{Authors' Contributions}

All authors have contributed to all parts of the article. All authors read and approved the final manuscript.

\section{References}

[1] Alegre, P., Blair, D. E. and Carriazo, A., Generalized Sasakian-space-forms, Israel J. Math., 14 (2004), 157-183.

[2] Alegre, P. and Carriazo, A., Submanifolds of generalized Sasakian-space-forms, Taiwanese J. Math., 13 (2009), 923-941.

[3] Alegre, P. and Carriazo, A., Structures on generalized Sasakian-space-forms, Diff. Geo. and its Application, 26 (2008), $656-666$.

[4] Alegre, P. and Carriazo, A., Generalized Sasakian-space-forms and conformal changes of the metric, Results in Math., 59 (2011), 485-493.

[5] Ashoka, S. R., Bagewadi, C. S. and Ingalahalli, G., Certain results on Ricci Solitons in $\alpha$-Sasakian manifolds, Hindawi Publ. Corporation, Geometry, Vol.(2013), Article ID 573925, 4 Pages.

[6] Ashoka, S. R., Bagewadi, C. S. and Ingalahalli, G., A geometry on Ricci solitons in $(\text { LCS })_{n}$-manifolds, Diff. Geom.-Dynamical Systems, 16 (2014), 50-62.

[7] Bagewadi, C. S. and Ingalahalli,G., Ricci solitons in Lorentzian-Sasakian manifolds, Acta Math. Acad. Paeda. Nyire., 28 (2012), 59-68.

[8] Bejan, C. L. and Crasmareanu, M., Ricci Solitons in manifolds with quasi-contact curvature, Publ. Math. Debrecen, 78/1 (2011), 235-243.

[9] Belkhelfa, M., Deszcz, R. and Verstraelen, L., Symmetry properties of generalized Sasakian-space-forms, Soochow J. Math., 31 (2005), 611-616.

[10] Blaga, A. M., $\eta$-Ricci solitons on para-kenmotsu manifolds, Balkan J. Geom. Appl., 20 (2015), 1-13.

[11] Blair, D. E., Contact manifolds in Riemannian geometry, Lecture Notes in Math., 509, Springer-Verlag, 1976.

[12] Carriazo, A., On generalized Sasakian-space-forms, Proceedings of the Ninth International Workshop on Diff. Geom., 9 (2005), 31-39.

[13] Chandra, S., Hui, S. K. and Shaikh, A. A., Second order parallel tensors and ricci solitons on $(L C S)_{n}$-manifolds, Commun. Korean Math. Soc., 30 (2015), 123-130.

[14] Chen, B. Y. and Deshmukh, S., Geometry of compact shrinking Ricci solitons, Balkan J. Geom. Appl., 19 (2014), 13-21.

[15] Deshmukh, S., Al-Sodais, H. and Alodan, H., A note on Ricci solitons, Balkan J. Geom. Appl.,16 (2011), 48-55.

[16] Ghefari, R. A., Al-Solamy, F. R. and Shahid, M. H., CR-submanifolds of generalized Sasakian-space-forms, JP J. Geom. and Topology, 6 (2006), 151-166.

[17] Gherib, F. and Belkhelfa, M., Second order parallel tensors on generaized Sasakian-space-forms and semi parallel hypersurfaces in Sasakian-space-forms, Beitrage Zur Algebra and Geom., 51 (2010), 1-7.

[18] Gherib, F., Gorine, M. and Belkhelfa, M., Parallel and semi symmetry of some tensors in generalized Sasakian-space-forms, Bull. Trans. Univ. Brasov, Series III: Mathematics, Informatics, Physics, 1(50) (2008), 139-148.

[19] Hamilton, R. S., Three-manifolds with positive Ricci curvature, J. Diff. Geom., 17 (1982), 255-306.

[20] Hamilton, R. S., The Ricci flow on surfaces, Mathematics and general relativity, Contemp. Math., 71, American Math. Soc., 1988, 237-262. 
[21] He, C. and Zhu, M., Ricci solitons on Sasakian manifolds, arxiv:1109.4407V2, [Math DG], (2011).

[22] Hui, S. K. and Chakraborty, D., Some types of Ricci solitons on (LCS)n-manifolds, J. Math. Sci. Advances and Applications, 37 (2016), 1-17.

[23] Hui, S. K., Lemence, R. S. and Chakraborty, D., Ricci solitons on three dimensional generalized Sasakian-space-forms, Tensor Society, N. S., 76 (2015).

[24] Hui, S. K. and Prakasha, D. G., On the C-Bochner curvature tensor of generalized Sasakian-space-forms, Proc. Natl. Acad. Sci., India, Sec. A, Phys. Sci., Springer, 85(3) (2015), 401-405.

[25] Hui, S. K. and Sarkar, A., On the W2-curvature tensor of generalized Sasakian-space-forms, Math. Pannonica, 23 (2012), 1-12.

[26] Ingalahalli, G. and Bagewadi, C. S., Ricci solitons in $\alpha$-Sasakian manifolds, ISRN Geometry, Vol.(2012), Article ID 421384, 13 Pages.

[27] Kim, U. K., Conformally flat generalized Sasakian-space-forms and locally symmetric generalized Sasakian-space-forms, Note di Matematica, 26 (2006), 55-67.

[28] Maralabhavi, Y. B. and Shivaprasanna, G. S., Second order parallel tensors on generalized Sasakian-space-forms, Int. J. Math. Eng. and Sci., 1 (2012), 11-21.

[29] Nagaraja, H.G. and Premalatta, C.R., Ricci solitons in Kenmotsu manifolds, J. Math. Analysis, 3(2) (2012), 18-24.

[30] Narain, D., Yadav, S. and Dwivedi, P. K., On generalized Sasakian-space-forms satisfying certain conditions, Int. J. Math. and Analysis, 3 (2011), 1-12.

[31] Olteanu, A., Legendrian warped product submanifolds in generalized Sasakian-space-forms, Acta Mathematica Academiae Paedagogice Nyiregyhaziensis, 25 (2009), 137-144.

[32] Patterson, E. M., Some theorems on Ricci-recurrent spaces, J. London Math. Soc., 27 (1952), 287-295.

[33] Perelman, G., The entropy formula for the Ricci flow and its geometric applications, http://arXiv.org/abs/math/0211159, 2002, $1-39$.

[34] Perelman, G., Ricci flow with surgery on three manifolds, http://arXiv.org/abs/math/0303109, 2003, 1-22.

[35] Pigola, S., Rigoli, M., Rimoldi, M. and Setti, A. G., Ricci almost solitons, Ann. Sc. Norm. Super. Pisa Cl. Sci, 10 (2011), 757-799.

[36] Pokhariyal, G. P. and Mishra, R. S., The curvature tensor and their relativistic significance, Yokohoma Math. J., 18 (1970), 105108.

[37] Shaikh, A. A. and Hui, S. K., Some global properties of pseudo cyclic Ricci symmetric manifolds, Applied Sciences, Balkan Soc. of Geom., 13 (2011), 97-101.

[38] Shaikh, A. A. and Hui, S. K., On 申-symmetric generalized Sasakian-space-forms admitting semi-symmetric metric connection, Tensor, N. S., 74 (2013), 265-274.

[39] Sharma, R., Certain results on $k$-contact and $(k, \mu)$-contact manifolds, J. Geom., 89 (2008),138-147.

[40] Shukla, S. S. and Chaubey, P. K., On invariant submanifolds in generalized Sasakian-space-forms, J. Dynamical Systems and Geometric Theories, 8 (2010), 173-188.

[41] Szabó, Z. I., Structure theorems on Riemannian spaces satisfying $R(X, Y) \cdot R=0$, The local version, J. Diff. Geom., 17 (1982), 531-582.

[42] Tripathi, M. M., Ricci solitons in contact metric manifolds, arxiv:0801,4221 V1, [Math DG], (2008).

[43] Yadav, S., Suthar, D. L. and Srivastava, A. K., Some results on $M\left(f_{1}, f_{2}, f_{3}\right)_{2 n+1}$-manifolds, Int. J. Pure and Appl. Math., 70 (2011), 415-423. 